\title{
Study of Project Development based on UC/OS- II Embedded System
}

\author{
Weipeng $\mathrm{Yao}^{1, \mathrm{a}}$ \\ ${ }^{1}$ Xi'an aeronautical university, Xi'an, Shaanxi, 710077 \\ ${ }^{\mathrm{a}}$ email
}

Keywords: Processor, Project Development, The Kernel

\begin{abstract}
With the world's first microcomputer from the date of birth, the embryonic development of embedded systems will have until today has been almost everywhere. Embedded systems development projects have a lot of paper on the concept and development status of embedded systems for a simple narrative, and pass on the UC/OS-II features introduced with the description, learn based on UC/OS-II embedded system project the basic process development.
\end{abstract}

\section{Introduction}

Currently, embedded system project development, there are many mature RTOS, such as Windows CE, Linux, UC/OS-II, UC/OS-III, etc., and UC/OS-II is a completely free, full source code disclosed, the basic functions of a stable operating system kernel. So, whether in commercial project development, or education and teaching, UC/OS-II is a good real-time operating system

\section{A Basic Overview of the Embedded System}

A dedicated embedded system of the computer system for a specific application, and a control device that is fully embedded inside the system. Because the embedded system to perform a task targeted and limitations, so the design is easier to optimize, reduce production costs. Core embedded system components are: one or more pre-programmed to execute several tasks minority microprocessor or microcontroller. Software on embedded systems are often referred to as "firmware", as compared with software on a general purpose computer is no variability, the user does not selectively.

\section{The Status of Development of Embedded Systems}

Embedded systems originated in the era of the microcomputer, the most basic way to understand it is to a microcomputer embedded in a target system in order to achieve the object of intelligent systems. In the digital age of today, the embedded system has achieved the computer hardware and software technology with a variety of technology integration level, broad range of applications. For example: smart phones, smart robots, aviation, marine, car navigation and so on. Since the embedded system is divided into two: one is a stand-alone embedded systems; another network embedded systems. With the application requirements and continuous research, namely the future of embedded systems from a single, intelligent network, standardization, integration and other comprehensive development.

\section{UC/OS-II Introduction and Features}

UC/OS-II a real-time operating system kernel, and has a cropped, easy real-time, easy to streamline other can optimize performance. The total amount of 200 lines of assembly language is reduced to a minimum, for the preparation of the associated UPC codes, most of the other code is written in C language. The main purpose is to be the development of embedded systems design. UC/OS-II with priority preemptive real-time way to the target, to achieve the purpose OS, then OS-based systems provide the appropriate service. Such as event logo, message queue, mutexes.

UC/OS-II since the development has been more than the carrier, has long been widely applied to 
various related fields, based on the unique nature and powerful features, in-depth the majority of embedded systems developers favor and respected. Because the characteristics of UC/OS-II in the range include the following five severely features:

From UC/OS-II has been able to achieve widespread application, simply because it allows different implantation on different hardware platforms, ultimately depend on openness UC/OS-II source code with other commercial real-time kernel full disclosure unmatched features.

UC/OS-II implantable strong, because UC/OS-II uses C language to write most of the source code, only a small portion of CPU-related written in assembly language, which makes the UC/OS-II CPU provides a variety of implantable regard, it can not only run on 8-bit, 16-bit, 32-bit CPU, can also run on 64-bit and DSP.

UC/OS-II highly curable, because UC/OS-II is specifically designed for embedded applications, so developers manage cured by any means, UC/OS-II can be embedded in the developer products.

UC/OS-II can be cut and strong, when UC/OS-II to improve service, users lose the excess part according to needs, leaving only the critical part, thereby reducing the UC/OS-II in the product memory Occupancy rate.

UC/OS-II has a characteristic and preemptive multitasking, because UC/OS-II has a fully preemptive real-time, so the choice to run the task with the highest priority as the standard, does not have the ability to rotate the scheduling method. Multitasking refers, for example, UC/OS-II 64 management tasks, the system will retain the eight, other 56 tasks in a different order of priorities arranged to run.

\section{Platform to Build and Implement based on UC/OS-II Embedded System}

Construction of the platform is a prerequisite for the development of embedded development, UC/OS-II is just a kernel, it is to improve task scheduling, play a fundamental role in the software system, to develop the application, there should be a corresponding value operating system, this operation must be complete and practical, real-time multi-task embedded system, before the establishment of the embedded operating system, the first will be UC/OS-II ported to the CPU, and its extended can be achieved.

Processor C compiler to produce reentrant code, interrupt support, can open or close an interrupt, can accommodate a certain amount of data, hardware stack, etc. is carried UC/OS-II transplant prerequisites, only to meet these conditions before it can be transplanted . In addition, before making expansion, it must be guaranteed after the UC/OS-II is a microprocessor implanted into normal operation. Because there are a lot of the UC/OS-II porting various processor instance, so long as the modified existing porting code on it. Samsung is a company ARM7TDMI microprocessor, this paper will do as an example UC/OS-II transplant. The main purpose of transplantation focus on OS $\neg$ CPU.H, OS_CPU_A.ASM, OS_CPU_C.C these three documents, with over three rewritable data and parameter files to achieve.

After will be UC/OS-II, also carried on UC/OS-II expansion, so as to be an operating system more complete, more practical. UC/OS-II expansion include: the establishment of an external device driver and a file system. Mainly to establish an external device drivers in order to achieve the corresponding function, such as a graphical user interface functions, and other program interface functions and the like. A file system, mainly because of UC/OS-II does not provide file system. Open up space in the SDRAM UC/OS-II alone is conducive to upload storage, transmission and application data. Specific extensions schematically in Figure 1. 


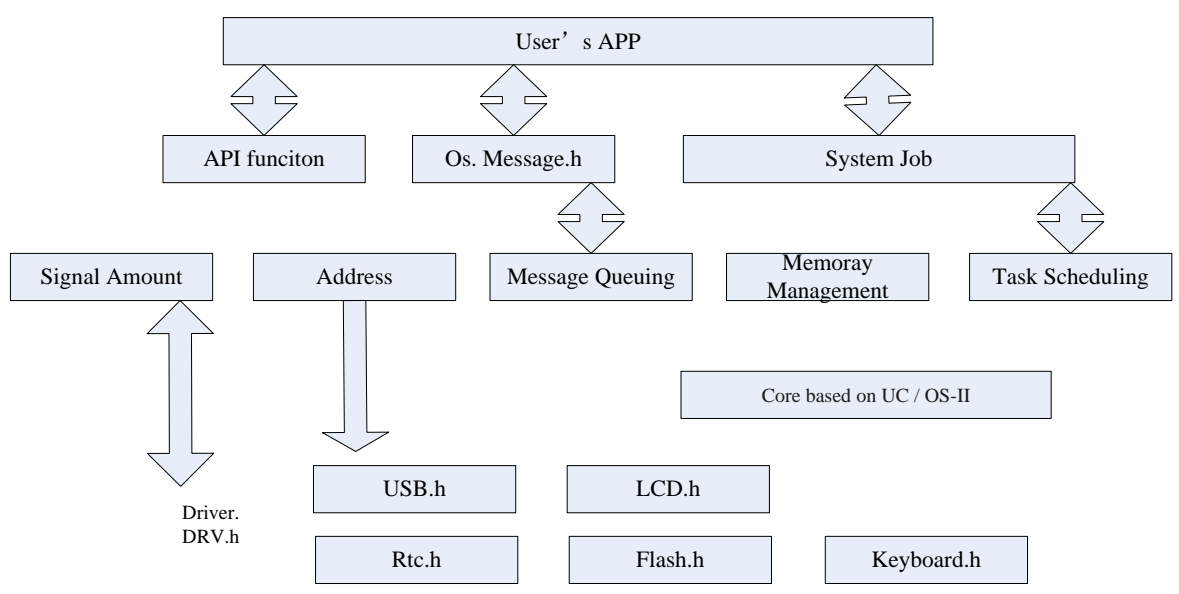

Fig.1 Based on UC/OS-II extended a schematic diagram

\section{Embedded System Project Development based on UC/OS- II}

UC/OS-II is mounted to the case of the $\mathrm{C}$ drive of a new project, a new project in C:ISOFTWARE $\backslash U$ cos-II, with a New Name, C:ISOFTWARE $\backslash$ Ucos-IINew file, C: $\backslash S O F T W A E \backslash U$ cos-II $\backslash$ ex2_x86I $\backslash B C 45$ has two folders, the two folders to copy all of the new directory in the new, the new project to rewrite the code, but note that you want to write SOURCEITEST.C carried out. The next step is to configure TEST some parts of the document to be changed.

SOURCE\INCLUDES.H changes to the file, as follows:

\#i nclude <string.h>

\#i nclude $<$ ctype.h $>$

\#i nclude $<$ stdlib.h $>$

\#i nclude $<$ conio.h $>$

\#i nclude $<$ dos.h $>$

\#i nclude < setjmp.h>

\#i nclude "\softwarelucos-iilix86l\bc45\os_cpu.h"

\#i nclude "os_cft.h"

\#i ncludelsoftwrelucos-iilsourcelucos_ii."

\#i nclude "\ softwarclblocks\pclbc45\pc.h"

This installation development tools and software packages does not matter, mainly based on the operational configuration file OS_CFC.H project.

Set of development tools that links the modules and parameters settings, set by turning on TEST.LNK configuration with an editor, and make editorial changes.

BorlandC the makefile file TEST.MAK be modified.

MAKETEST.BAT configure, build $\backslash L S T, I W O R K, I O B J$ directory in MAKETEST.BAT three, and with the same name and path TEST.MAK, then Copy thelTEST directory to TEST.MAKIWORK directory, and let BC's makefile program execution, automatic compilation, automatic links, but C: path problem BC45 must pay attention to.

The development of related programs can be carried out, mainly to prepare Test.c file, when the development projects have multiple source files, and do not need to change the settings for each of the eleven files, just Test.c with \#include Therefore, the source file that contains the statement can be.

In the program\SOFTWARE\BLOCKS\SAMPLE\SOURCE\TEST run, you must compile a makefile, then the call to be successful by executing MAKETEST.BAT.

\section{Conclusion}

Embedded systems have long been widely used, can be seen everywhere in modern life, such as military equipment, commercial facilities, personal living facilities. Development UC/OS-II 
embedded system project based on the premise that to grasp the UC/OS-II application foundation and development process, only to lay a solid foundation of knowledge, based on a deep understanding of UC/OS-II embedded system project development based processes, the actual development work can be more handy.

\section{References}

[1] Yang Li. realized on ucos- II embedded operating system platform exfat file system [J]. Science and Technology Information, 2015, 13 (5): 11.

[2] Chen Jin, Ma Shangchang, Zhangsu Juan. aResearch-based networking technology UCOS- II and LwIP serial devices [J]. Electronic Design Engineering, 2015, 23 (10): 75-78.

[3] Rowebots, Kim Rowe, Zhang Aihua, etc. Chapter 6: Based Things secure embedded operating system [J]. Microcontroller and Embedded Systems Applications, 2015, 15 (1): 75-77. 